PROCEEDINGS OF THE AMERICAN MATHEMATICAL SOCIETY

Volume 124, Number 9, September 1996

\title{
FORCING OF PERIODIC ORBITS FOR INTERVAL MAPS AND RENORMALIZATION OF PIECEWISE AFFINE MAPS
}

\author{
MARCO MARTENS AND CHARLES TRESSER \\ (Communicated by Linda Keen)
}

\begin{abstract}
We prove that for continuous maps on the interval, the existence of an $n$-cycle implies the existence of $n-1$ points which interwind the original ones and are permuted by the map. We then use this combinatorial result to show that piecewise affine maps (with no zero slope) cannot be infinitely renormalizable.
\end{abstract}

\section{INTRODUCTION}

A fascinating feature of real analytic infinitely renormalizable interval maps is that their attracting invariant Cantor sets seem to have a complicated geometry (for the unimodal case, see for instance [S] and references therein). One could hope to avoid this complexity by constructing piecewise affine examples (with no zero slope). This is indeed the case when there are infinitely many intervals of affinity (see e.g. $[\mathrm{T}]$ ), but we show in section 3 that no example exists with finitely many intervals of affinity. In order to prove these results we had to solve some questions about forcing of permutations which are described in section 2 .

We now state our main results. The collection of continuous maps on the interval is denoted by $C^{0}([0,1])$. A cycle of a map $f \in C^{0}([0,1])$ is a collection of pairwise disjoint closed intervals $\mathcal{I}=\left\{I_{0}, I_{1}, \ldots, I_{q-1}\right\}$ which are cyclically permuted by $f$. That means $f\left(I_{i}\right) \subset I_{i+1 \bmod q}$. The set $\bigcup_{l=0}^{q-1} I_{l}$ is denoted by $O(\mathcal{I})$. The cycle is called trivial if all intervals are points.

A cycle $\mathcal{I}^{j}$ refines a cycle $\mathcal{I}^{i}$ if $O\left(\mathcal{I}^{j}\right) \subset O\left(\mathcal{I}^{i}\right)$. In this situation there is a number $a_{i, j} \in \mathbb{N}$ such that every component of $\mathcal{I}^{i}$ contains $a_{i, j}$ components of $\mathcal{I}^{j}$. We will always assume $a_{i, j} \geq 2$ and use the notation $\mathcal{I}^{i} \supset \mathcal{I}^{j}$ or $\mathcal{I}^{j} \subset \mathcal{I}^{i}$.

Let $\mathcal{I}^{j}$ refine $\mathcal{I}^{i}$. A connected component $G$ of $O\left(\mathcal{I}^{i}\right) \backslash O\left(\mathcal{I}^{j}\right)$ is called a gap if $\partial G \subset O\left(\mathcal{I}^{j}\right)$. The union of gaps is denoted by $\mathcal{G}\left(\mathcal{I}^{i}, \mathcal{I}^{j}\right)$.

Definition 1.1. Let $f \in C^{0}([0,1])$, and let $\mathcal{I}^{2}$ be a cycle which refines the cycle $\mathcal{I}^{1}$. An invariant set $P$ of periodic points of $f$ is called a splitting of the pair of cycles $\mathcal{I}^{1} \supset \mathcal{I}^{2}$ if

- $P \subset \mathcal{G}\left(\mathcal{I}^{1}, \mathcal{I}^{2}\right)$

- every gap in $\mathcal{G}\left(\mathcal{I}^{1}, \mathcal{I}^{2}\right)$ contains exactly one point of $P$.

Theorem A. Every pair of cycles $\mathcal{I}^{1} \supset \mathcal{I}^{2}$ of a map $f \in C^{0}([0,1])$ admits a splitting.

Received by the editors December 29, 1994.

1991 Mathematics Subject Classification. Primary 58F11.

(C)1996 American Mathematical Society 
Theorem A is a main ingredient of the proof of Theorem C below. It is a corollary of the following result.

Theorem B. If a continuous map $f$ on the interval has a periodic orbit of period $n$, then it permutes $n-1$ points interwinding the periodic orbit.

Here we say that a set of $n-1$ points on the real line interwinds a set of $n$ points if any two consecutive points of any of these sets are separated by a point of the other set. Theorem B in turns follows from a property of markov maps induced by permutations of points in the interval, stated as Theorem 2.1 .

A map $f \in C^{0}([0,1])$ is infinitely renormalizable if it has an infinite sequence of refining cycles

$$
\mathcal{I}^{1} \supset \mathcal{I}^{2} \supset \mathcal{I}^{3} \supset \cdots .
$$

The set $\bigcap O\left(\mathcal{I}^{n}\right)$ is called an infinitely renormalizable invariant set. A map $f \in C^{0}([0,1])$ is called piecewise affine if there is a sequence of points $0=c_{-1}<$ $c_{0}<\cdots<c_{d}<c_{d+1}=1$, called corner points, such that $f$ is affine with non-zero slope on each interval $\left[c_{i}, c_{i+1}\right]$ and $f$ is not affine on any larger interval. The set of corners of $f$ is denoted by $C_{f}$. The collection of all piecewise affine maps is denoted by $P L([0,1])$.

Theorem C. There are no infinitely renormalizable piecewise affine maps.

Notice that the conclusion of Theorem $\mathrm{C}$ fails if $C_{f}$ is allowed to be countable (see e.g. $[\mathrm{T}]$ ) or if we relax the non-zero slope condition (see e.g. [BMT]). Theorem C can be understood as a step toward proving the conjecture in [GMT], that piecewise affine maps on the interval without periodic attractors are eventually expanding. Observe that this Conjecture and Theorem $\mathrm{C}$ hold trivially for the well-known tent-maps.

An important ingredient of the proof of Theorem C is the Expansion-Lemma in section 3. It states that there is a reasonable big collection of expanding periodic orbits with exponent away from 0. This Expansion-Lemma should be compared with the Finiteness of Attractors Theorem in [MMS], which states that in smooth maps, periodic orbits with sufficiently high period are expanding with exponent away from 0 . Whether this is also true for piecewise affine maps is part of the conjecture in $[\mathrm{GMT}]$.

\section{FORCING}

Let $S_{n}$ be the collection of permutations of $N_{n}=\{1,2, \ldots, n\}$. For every $\pi \in S_{n}$ we define the $(n-1) \times(n-1)$-matrix $F_{\pi}$, with

- $F_{\pi}(i, j)=1$, if $(j, j+1) \subset(\pi(i), \pi(i+1))$,

- $F_{\pi}(i, j)=0$ otherwise.

Observe that the subshift of finite type defined by the matrix $F_{\pi}$ corresponds to the piecewise affine interpolation of the permutation $\pi$.

A continuous map $f:[1, n] \rightarrow[1, n]$ with $f \mid N_{n}=\pi$ will in general map the gaps $(i, i+1)$ in a very non-monotone way, and one cannot guess the full set of periodic orbits of $f$ by only the knowledge of $\pi$. However $f \mid N_{n}=\pi$ implies some minimal complexity for the dynamics of $f$. More specifically, it is known that the subshift of finite type defined by $F_{\pi}$ can always be monotonically imbedded into the dynamics of $f$; we say that $\pi=f \mid N_{n}$ forces all the dynamics of the subshift defined by $F_{\pi}$. In the above statement, monotonicity refers to the skewed lexicographic order on 
the sequence space, as used in kneading theory (see e.g. [MT]), and the usual order on $[1, n]$. As usual we identify the matrix $F_{\pi}$ with the corresponding subshift.

If $\phi \in S_{m}$ and $F$ is an $m \times m$-matrix with 0,1 entries, such that $F(i, \phi(i))=1$, we say that $F$ contains the permutation $\phi$, and write $\phi \ll F$.

Theorem 2.1. For every $\pi \in S_{n}, n \geq 2$, there exists a $\phi \in S_{n-1}$ with

$$
\phi \ll F_{\pi} \text {. }
$$

Remark. It is easy to construct examples of subshifts of finite type whose defining matrices do not contain any permutation but have some power with positive entries. These examples let Theorem 2.1 appear as a specific property of subshifts of finite type defined by permutations.

The proof of this Theorem needs some preparation. We are going to describe a "cutting" procedure on permutations and a related "cutting" procedure on their matrices. Let $n \geq 2$ and $k \leq n-1$, and let $j_{k}: N_{n-1} \rightarrow N_{n} \backslash\{k\}$ stand for the order preserving bijection. If $\pi \in S_{n}$ and $k=\pi^{-1}(n)$, then $\hat{\pi} \in S_{n-1}$ is defined by

$$
\hat{\pi}(i)=\pi\left(j_{k}(i)\right)
$$

where $i \in N_{n-1}$.

From the definition of the matrix $F_{\pi}$, the 1's are consecutive in each of its rows. Furthermore if $F_{\pi}(k, n)=1$, then $\pi(k)=n$ or $\pi(k+1)=n$. So there are at most two rows with the last entry equal to 1 , and such rows have to be consecutive.

Consider the rows with last entry equal to 1 and assume that row $k$ has the shortest block of 1's among those: that means, if $F_{\pi}(l, n)=1$ and $l \neq k$, then there exists $j \leq n$ with $F_{\pi}(l, j)=1$ and $F_{\pi}(k, j)=0$. Now we define an $(n-1) \times(n-1)$ matrix $\widehat{F}_{\pi}$ by

$$
\widehat{F}_{\pi}(i, j)=F_{\pi}\left(j_{k}(i), j\right) .
$$

This ^-operation on matrices has been defined for matrices induced by permutations. To the contrary of the ${ }^{\text {N}}$-operation on permutations, in general it cannot be squared because the new matrix is maybe not induced by a permutation (this would always be the case for permutations realizable as restrictions of unimodal maps). In particular $F_{\hat{\pi}}$ is in general not equal to $\widehat{F}_{\pi}$. These two matrices are only equal for unimodal permutations. In general we have

Proposition 2.2. For every $\pi \in S_{n}, n \geq 2$,

$$
F_{\hat{\pi}} \leq \widehat{F}_{\pi} .
$$

Proof. Fix $\pi \in S_{n}$. For $k \leq n-1$ let $h_{k}: N_{n-2} \backslash\{k-1\} \rightarrow N_{n-1} \backslash\{k-1, k\}$ be the order preserving bijection.

If $\pi^{-1}(n)=k$, then the $(k-1)^{\text {th }}$ and $k^{\text {th }}$ row have a 1 in their last entry. Denote these rows by the vectors $V$ and $v$. Assume that $V$ has a longer block of 1 's. In the case that $k=1$ or $k=n$ there is only one row whose last entry equals one, respectively the first or the last row. In these cases let $V$ be this row and $v=0$.

Now the Proposition follows immediately from

Claim. If $i \neq k-1$, then

$$
\begin{aligned}
& F_{\hat{\pi}}(i, j)=F_{\pi}\left(h_{k}(i), j\right), \\
& \widehat{F}_{\pi}(i, j)=F_{\pi}\left(h_{k}(i), j\right) .
\end{aligned}
$$


For $j \leq n-2$ and $k \neq 1, n$

$$
\begin{aligned}
& F_{\hat{\pi}}(k-1, j)=V(j)-v(j), \\
& \widehat{F}_{\pi}(k-1, j)=V(j) .
\end{aligned}
$$

Proof. The matrix $\widehat{F}_{\pi}$ was obtained from $F_{\pi}$ by erasing the last column and row $v$. The result of this operation is expressed in the claim.

Now consider the $\hat{\pi}$ image of the gap $(i, i+1)$. If $i \leq k-2$, then the $\hat{\pi}$-image is the same as the $\pi$-image. Hence the first $k-2$ rows of $F_{\hat{\pi}}$ equal the first $k-2$ rows of $F_{\pi}$. If $i \geq k$, then the $\hat{\pi}$-image is the $\pi$-image of the gap $(i+1, i+2)$. So the last $(n-2)-(k-1)$ rows of $F_{\hat{\pi}}$ equal the last $(n-1)-k$ rows of $F_{\pi}$. These properties are expressed in

$$
F_{\hat{\pi}}(i, j)=F_{\pi}\left(h_{k}(i), j\right),
$$

whenever $i \neq k-1$. It just remains to consider the image of the $(k-1)^{\text {th }}$ gap. The boundary points are $k-1$ and $k$. Hence the $\hat{\pi}$-image of this gap equals the interval $(\pi(k-1), \pi(k+1))$. It follows easily that if a gap $(j, j+1)$ is covered by the interval $(\pi(k-1), \pi(k+1))$, then $V(j)-v(j)=1$.

Proof of Theorem 2.1. The proof of Theorem 2.1 is by induction. For a permutation $\pi \in P_{S}$ we have that the only entry of $F_{\pi}$ equals 1 . Hence $F_{\pi}$ contains a permutation. Now suppose that every matrix $F_{\pi}$ with $\pi \in S_{n}$ contains a permutation.

Let $\pi \in S_{n+1}$. Then $\hat{\pi} \in S_{n}$ and hence $F_{\hat{\pi}}$ contains a permutation $\phi \ll F_{\hat{\pi}}$. From Proposition 2.2 we get $F_{\hat{\pi}} \leq \widehat{F}_{\pi}$. Hence $\phi_{0} \ll \widehat{F}_{\pi}$.

Say that $\widehat{F}_{\pi}$ was obtained by cutting the $k^{\text {th }}$ row of $F_{\pi}$. Then $F_{\pi}$ contains the permutation $\phi \in S_{n+1}$ defined by

$$
\begin{aligned}
\phi(k) & =n, \\
\phi(i) & =\phi_{0}\left(j_{k}^{-1}(i)\right) \text { if } i \neq k .
\end{aligned}
$$

Proof of Theorem B. Let $f$ be a continuous map with $\left\{p_{1}, p_{2}, \ldots, p_{n}\right\}$ a periodic orbit of period $n$. Assume $p_{1}<p_{2}<\cdots<p_{n}$, and let $G_{i}=\left(p_{i}, p_{i+1}\right)$, with $i=1,2, \ldots, n-1$. By Theorem 2.1 we know that there exists a permutation $\phi \in S_{n-1}$ and intervals $T_{i} \subset G_{i}$ such that $f: T_{i} \rightarrow G_{\phi(i)}$ is onto. The continuity of $f$ assures the existence of points $s_{i} \in \overline{T_{i}}$ such that $f\left(s_{i}\right)=s_{\phi(i)}$, with $i=1,2, \ldots, n-1$. Clearly the points $s_{i}$ are periodic with period less than $n$. In particular, $s_{i} \in \operatorname{int}\left(G_{i}\right)$; they actually interwind the original orbit.

Proof of Theorem A. To prove Theorem A we may collapse the cycle $\mathcal{I}^{2}$ into a periodic orbit, say $\mathcal{I}^{2}=\left\{p_{1}, p_{2}, \ldots, p_{q_{2}}\right\}$. Again, as in the proof of Theorem B, let $G_{i}=\left(p_{i}, p_{i+1}\right)$. Apply Theorem 2.1 to get a permutation $\phi \in S_{q_{2}-1}$ and the intervals $T_{i} \subset G_{i}$ such that $f: T_{i} \rightarrow G_{\phi(i)}$ is onto. Let $P^{\prime}=\left\{s_{1}, s_{2}, \ldots, s_{q_{2}-1}\right\}$ be the corresponding points which interwind $\left\{p_{1}, p_{2}, \ldots, p_{q_{2}}\right\}$, that is, $s_{i} \in T_{i}$ and $f\left(s_{i}\right)=s_{\phi(i)}$.

Unfortunately $P^{\prime}$ will not be a splitting for the pair $\mathcal{I}^{2} \subset \mathcal{I}^{1}$; it contains also points outside $\mathcal{I}^{1}$. Let $P=P^{\prime} \cap G\left(\mathcal{I}^{1}, \mathcal{I}^{2}\right)$. To show that $P$ is a splitting it suffices 
to show that $P$ is invariant. Take $s_{i} \in P$. Then $T_{i}$ is a subset of the cycle $\mathcal{I}^{1}$. Hence $f\left(T_{i}\right)=G_{\phi(i)}$ is a subset of the cycle $\mathcal{I}^{1}$; in particular, $G_{\phi(i)}$ is a gap. So $s_{\phi(i)} \in G\left(\mathcal{I}^{1}, \mathcal{I}^{2}\right) \cap P^{\prime}=P$.

\section{Renormalization}

In this section we are going to prove Theorem $\mathrm{C}$. The main reason why piecewise affine maps cannot be infinitely renormalizable is the fact that there are enough periodic orbits with some definite expansion.

A cycle $\mathcal{I}^{2}$ is called a doubling of a cycle $\mathcal{I}^{1}$ if $\mathcal{I}^{1} \supset \mathcal{I}^{2}$ and $a_{1,2}=2$.

Expansion-Lemma 3.1. Let $f \in P L([0,1])$ having two cycles $\mathcal{I}^{1} \supset \mathcal{I}^{2}$ with splitting P. If

- $\mathcal{I}^{2}$ is not a doubling of some $\mathcal{I} \subset \mathcal{I}^{1}$ and

- $C_{f} \subset O\left(\mathcal{I}^{2}\right)$,

then there exists $x \in P$ with

$$
\left|D f^{p}(x)\right| \geq 1+e^{-11 V}
$$

where $p$ is the period of $x$ and $V$ the variation of $\log (|D f|)$.

Proof. Let $I \in \mathcal{I}^{1}$, and let $G \subset I$ be a gap, say $\partial G \subset I_{l}^{2} \cup I_{r}^{2}$. Now there exists a periodic point $x \in P \cap G$. Say it has period $p \in \mathbb{N}$.

Claim 1. There exists a gap $G^{\prime} \subset I$ and $G^{\prime} \neq G$ and an interval $K \subset G$ with $f^{p}: K \rightarrow G^{\prime}$ onto.

Proof of Claim 1: Say $\mathcal{I}^{2}$ has $q_{2}$ intervals. So $p \leq q_{2}-1$. Assume Claim 1 is not true; then $f^{p}(G) \subset I_{l}^{2} \cup G \cup I_{r}^{2}$ and $f^{p}\left(I_{l}^{2} \cup I_{r}^{2}\right) \subset I_{l}^{2} \cup I_{r}^{2}$. Because $p<q_{2}$, the two intervals $I_{l}^{2}$ and $I_{r}^{2}$ have to be interchanged. In particular $p=\frac{1}{2} q_{2}$. Let $H=$ $I_{l}^{2} \cup G \cup I_{r}^{2}$; then $f^{p}(H) \subset H$. If we can show that the orbit $\left\{H, f(H), \ldots, f^{p-1}(H)\right\}$ is pairwise disjoint, then we would have shown that $\mathcal{I}^{2}$ is a doubling of some cycle $\mathcal{H} \subset \mathcal{I}^{1}$. This contradicts the assumptions and Claim 1 would be proved.

So suppose $f^{j}(H) \cap f^{p}(H) \neq \varnothing$, for some positive $j \leq p-1$. Now $I_{l+j}^{2}$ and $I_{r+j}^{2}$ do not intersect $H$, since only $I_{l}^{2}$ and $I_{r}^{2}$ intersect $H$. Assume with no loss of generality that $I_{l}^{2} \subset f^{j}(H)$. This implies $I_{l+p-j}^{2}=f^{p-j}\left(I_{l}^{2}\right) \subset f^{p}(H) \subset H$. This is impossible because only $I_{l}^{2}$ and $I_{r}^{2}$ are in $H$. We proved that the orbit of $H$ forms a cycle. Claim 1 is proved.

Remark: In Claim 1, $G^{\prime}$ can be considered to be a gap adjacent to $I_{l}^{2}$ or $I_{r}^{2}$.

Now let $T \subset G$ be the maximal interval containing $x$ such that $\left(f^{p}(T) \cap O\left(\mathcal{I}^{2}\right)\right) \subset$ $I_{l}^{2} \cup I_{r}^{2}$. Using Claim 1 we find an interval $K \subset T$ with $f^{p}: K \rightarrow G^{\prime}$ onto. Because all corner points and their orbits are in $O\left(\mathcal{I}^{2}\right)$, the map $f^{p}: K \rightarrow G^{\prime}$ is in fact affine and onto. A collection of intervals in $[0,1]$ is said to have intersection multiplicity $w$ if every point in $[0,1]$ is contained in at most $w$ intervals of the collection.

Claim 2. The intersection multiplicity of $\left\{T, f(T), f^{2}(T), \ldots, f^{p-1}(T)\right\}$ is at most 11. In particular

$$
\operatorname{Var}\left(\log \left(\left|D f^{p}\right| T \mid\right)\right) \leq 11 V .
$$

Proof of Claim 2: To prove Claim 2 it is enough to show that there are at most 10 values $1 \leq i \leq p-1$ such that $f^{i}(T) \cap f^{p}(T) \neq \varnothing$.

Let $I_{L}^{2}$ be the left neighbor of $I_{l}^{2}$ and $I_{R}^{2}$ be the right neighbor of $I_{r}^{2}$. The interval between $I_{L}^{2}$ and $I_{R}^{2}$ is denoted by $S$. Clearly $f^{p}(T) \subset S$. Observe that $S$ contains at most 3 gaps. Because the orbit of $x$ is one of the splitting periodic orbits in 
$P$, the orbit of $x$ intersects $S$ in at most three points, say in $x, f^{a}(x)$ and $f^{b}(x)$. These three intersections can also give rise to an intersection of $f^{a}(T)$ or $f^{b}(T)$ with $f^{p}(T)$.

Consider an intersection $f^{i}(T) \cap f^{p}(T) \neq \varnothing$, where $i \leq p-1$ and $i \notin\{0, a, b\}$. Because $f^{i}(x) \notin I_{L}^{2} \cup S \cup I_{R}^{2}$, the interval $I_{L}^{2}$ (or $I_{R}^{2}$ ) is contained in $f^{i}(T)$. Hence $L+p-i \in\left\{l, r, l+q_{2}, r+q_{2}\right\}$ (or $R+p-i \in\left\{l, r, l+q_{2}, r+q_{2}\right\}$ ). This means that there are at most 8 possible values for $i \notin\{0, a, b\}$ giving rise to an intersection.

All together we get at most $2+4+4=10$ intersections $f^{i}(T) \cap f^{p}(T) \neq \varnothing$, with $1 \leq i \leq p-1$. The intersection multiplicity is at most 11 . Claim 2 is proved.

Because the orbits of the corners are contained in $O\left(\mathcal{I}^{2}\right)$ there exists an interval $D \subset T$ with $x \in D$ and $f^{p}: D \rightarrow G$ affine and onto.

To prove Lemma 3.1, assume that $G$ was chosen the smallest gap in $I,|G| \leq\left|G^{\prime}\right|$. Furthermore $|K| \leq|T|-|D|$. Observe $\frac{|G|}{|D|}=\left|D f^{p}(x)\right|$ and $\frac{\left|G^{\prime}\right|}{|K|}=\left|D f_{\mid K}^{p}\right|$. Then

$$
\begin{aligned}
11 V & \geq \log \left(\left|D f^{p}\right| K \mid\right)-\log \left(\left|D f^{p}(x)\right|\right) \\
& =\log \left(\frac{\left|G^{\prime}\right|}{|K|} \times \frac{|D|}{|G|}\right) \\
& \geq \log \frac{|D|}{|K|} \geq \log \frac{|D|}{|T|-|D|} .
\end{aligned}
$$

This implies

$$
\left|D f^{p}(x)\right|=\frac{|G|}{|D|} \geq \frac{|T|}{|D|} \geq 1+e^{-11 V}
$$

Proof of Theorem C. The proof will be given in $P L$. This is the collection of piecewise affine maps defined on a finite union of intervals. The proof of Theorem $\mathrm{C}$ will be by contradiction. Suppose that $P L([0,1])$ has an infinitely renormalizable map. Then also $P L$ has an infinitely renormalizable map. Let $f \in P L$ be an infinitely renormalizable map, whose number of corner points is minimal. Denote the cycles by

$$
\mathcal{I}^{1} \supset \mathcal{I}^{2} \supset \mathcal{I}^{3} \supset \cdots
$$

Use the notation $q_{n}=\# \mathcal{I}^{n}$. We may assume that this sequence of cycles is complete. This means that if there is some cycle $\mathcal{I}$ with $\mathcal{I}^{n+1} \subset \mathcal{I} \subset \mathcal{I}^{n}$, then $\# \mathcal{I}=q_{n}$ or $\# \mathcal{I}=q_{n+1}$. It can be shown that the infinitely renormalizable invariant set is a minimal Cantor set (this follows from the non-existence of wandering intervals for maps in $P L$ ). The minimality of the action of $f$ on its infinitely renormalizable Cantor set and the minimality of the number of corner points allow us to assume

Claim 1. $C_{f} \subset \operatorname{int}\left(O\left(\mathcal{I}^{n}\right)\right)$ for all $n \in \mathbb{N}$.

Let $V=\operatorname{Var}(\log (|D f|))$ and set $R_{n, j}=f^{q_{n}} \mid I_{j}^{n}, n \in \mathbb{N}$ and $j=0,1,2, \ldots, q_{n}-1$.

Claim 2. There exists $K \in \mathbb{R}$ such that for all $n \in \mathbb{N}, j<q_{n}$

$$
\left|D R_{n, j}(x)\right| \leq K
$$

for all $x \in I_{j}^{n}$.

Proof: Observe that $R_{n, j} \in P L\left(I_{j}^{n}\right)$ and that it has uniform, that is, independent of $n$ and $j$, bounds on the numbers of corner points. Furthermore

$$
\operatorname{Var}\left(\log \left(\left|D R_{n, j}\right|\right)\right) \leq V \text {. }
$$

Now Claim 2 follows easily. 
Let $P_{n}$ be a splitting for the pair $\mathcal{I}^{n} \supset \mathcal{I}^{n+1}$. Claim 1 allows us to define

$$
\begin{aligned}
B_{n} & =\sum_{x \in \partial\left(O\left(\mathcal{I}^{n}\right)\right)} \log (|D f(x)|), \\
M_{n} & =\sum_{x \in P_{n}} \log (|D f(x)|) .
\end{aligned}
$$

Claim 3. For every $n \in \mathbb{N}$

$$
B_{n+1}=B_{n}+2 M_{n}
$$

In particular,

$$
B_{n}=B_{1}+\sum_{k=1}^{n-1} 2 M_{n} .
$$

Proof: Consider $y \in \partial I_{j}^{n+1} \subset I_{i}^{n}$. If $y$ is also in the boundary of some gap $G$, then $D f(y)=D f(x)$, where $x \in P_{n} \cap G$. This equality holds because all corner points are in $O\left(\mathcal{I}^{n+1}\right)$. In the other case, if $y$ is not in the boundary of some gap, then $D f(y)=D f(z)$, where $z \in \partial I_{i}^{n}$. Again equality holds because $C_{f} \subset O\left(\mathcal{I}^{n+1}\right)$.

Observe that all boundary points of $O\left(\mathcal{I}^{n}\right)$ are counted once and all splitting periodic points twice. Claim 3 is proved.

Claim 4. For every periodic point $x$, with period $p, \log \left(\left|D f^{p}(x)\right|\right)>0$. In particular,

$$
M_{n}>0
$$

for all $n \in \mathbb{N}$.

Proof: It is easy to see that a periodic attractor attracts a corner point. Because $q_{n} \geq 2^{n-1} q_{1} \rightarrow \infty$ every periodic orbit falls eventually outside $O\left(\mathcal{I}^{n}\right)$. An attracting periodic orbit would take a corner point with it, contradicting the minimality of corner points of $f$. The non-existence of neutral periodic orbits follows by a similar argument.

By Claims 3 and 4 we know that the sequence $B_{n}$ increases.

Claim 5 .

$$
\lim _{n \rightarrow \infty} B_{n}=B<\infty .
$$

Proof: The corners of $f$ are denoted by $C_{f}=\left\{c_{1}, c_{2}, \ldots, c_{d}\right\}$. Take $x \in I_{0}^{n}$. Furthermore let $c_{j} \in I_{k_{j}}^{n}$ and $v_{j}=\log \left(\left|D f_{+}\left(c_{j}\right)\right|\right)-\log \left(\left|D f_{-}\left(c_{j}\right)\right|\right), j=1,2, \ldots, d$ (the + and - means right and left derivatives). From [MMS] we know that $f$ does not have wandering intervals. This implies that the length of the intervals in $\mathcal{I}^{n}$ tends to zero for $n \rightarrow \infty$. So (in $P L$ ) we may assume that every interval in $\mathcal{I}^{n}$ contains at most one corner point.

It is an easy exercise to compare $\log \left(\left|D f^{q_{n}}(x)\right|\right)$ with $B_{n}$. There exist numbers $\sigma_{n, j}(x) \in\{-1,1\}$ such that

$$
2 \log \left(\left|D f^{q_{n}}(x)\right|\right)=B_{n}+\sum_{j=1}^{d} \sigma_{n, j}(x) v_{j},
$$

where $\sigma_{n, j}(x)=1$ if and only if $f^{k_{j}}(x) \in I_{k_{j}}^{2}$ is on the right of $c_{j}$ (remember $c_{j}$ is the unique corner point in $I_{k_{j}}^{2}$ ). 
From Claim 2 we get a bound on $\left|D f^{q_{n}}(x)\right|$. The finite sum in the right hand side of the above equation is clearly uniformly bounded. Hence we get a uniform bound on $B_{n}$, and Claim 5 follows.

As a consequence we get

$$
\lim _{n \rightarrow \infty} M_{n}=0 .
$$

But now we can apply the Expansion-Lemma: eventually $\mathcal{I}^{n+1}$ is a doubling of $\mathcal{I}^{n}$, for all $n \geq n_{0}$. This means that the splitting $P_{n}$ becomes a single periodic orbit hitting every component of the cycle $\mathcal{I}^{n}$ exactly once.

As above we get the existence of numbers $\sigma_{n, j} \in\{-1,1\}$ such that

$$
2 M_{n}=B_{n}+\sum_{j=1}^{d} \sigma_{j}^{n} v_{j} .
$$

Because $M_{n} \rightarrow 0$ and $B_{n} \rightarrow B$ and the fact that the sum in the above equality takes only finitely many values we get eventually

$$
\sum_{j=1}^{d} \sigma_{j}^{n} v_{j}=-B .
$$

Combining this with $B_{n+1}=B_{n}+2 M_{n}$ we get

$$
B_{n+1}=2 B_{n}-B \text {. }
$$

For the sequence $B_{n}$ to be bounded we need $B_{n} \equiv B$. So $M_{n} \equiv 0$, contradicting Claim 4.

\section{ACKNOWLEDGEMENTS}

After proving Theorem $\mathrm{C}$ in the case of period doubling $\left(a_{i, i+1} \equiv 2\right)$ we checked with some colleagues about its originality. Some time later, Michal Misiurewicz and Karen Brucks reported to us that V. J. Lopez and L. Snoha had recently obtained the same result [LS].

\section{REFERENCES}

[BMT] K. M. Brucks, M. Misiurewicz, C. Tresser, Monotonicity properties of the family of trapezoidal maps, Commun. Math. Phys. 137 (1991), 1-12. MR 92e:58108

[GMT] R. Galeeva, M. Martens, C. Tresser, Inducing, Slopes, and Conjugacy Classes, preprint 1994/4 at SUNY at Stony Brook, Israel J. Math. (to appear).

[MMS] M. Martens, W. de Melo, S. van Strien, Julia-Fatou-Sullivan Theory for real onedimensional dynamics, Acta Math. 168 (1992), 273-318. MR 93d:58137

[MT] J. Milnor, W. Thurston, On iterated maps of the interval, Springer Lecture Notes in Mathematics 1342 (1988), 465-563. MR 90a:58083

[LS] V. J. Lopez, L. Snoha, to appear.

[S] D. Sullivan, Bounds, quadratic differentials and renormalization conjectures, Mathematics into the twenty-first Century: 1988 Centennial Symposium, ed. F. Browder, Amer. Math. Soc. (1992), 417-466. MR 93k:58194

[T] C. Tresser, Fine structure of universal Cantor sets, Instabilities and Nonequilibrium structures III, eds. E. Tirapegui and W. Zeller (Reidel) Dordrecht (1991). MR 93j:58045

Institute of Mathematical Sciences, SUNy at Stony Brook, Stony Brook, New York 11794-3651

E-mail address: marco@math.sunysb.edu

I.B.M., P.O. Box 218, Yorktown Heights, New York 10598

E-mail address: tresser@watson.ibm.com 